\title{
A review of "Random graphs and complex networks" by Hofstad
}

\author{
Ghulam Abbas* ${ }^{*}$
}

\section{${ }^{*}$ Correspondence:}

abbasg@giki.edu.pk

Faculty of Computer Science

and Engineering, GIK Institute

of Engineering Sciences

and Technology, Topi 23640,

District Swabi, Pakistan

\author{
Book details \\ Hofstad, R \\ Random Graphs and Complex Networks. vol. 1. \\ New York: Cambridge University Press; 2017. \\ 321 pages; ISBN: 978-1-107-17287-6
}

Keywords: Complex networks, Graph theory, Probability theory, Random graphs

\section{Overview}

A random graph is a collection of vertices and edges connecting pairs of them at random based on edge probabilities (Newman et al. 2001; Hatano and Mesbahi 2005). The theory of random graphs was introduced by Erdős and Rényi (1960) to give a probabilistic construction of a graph with large girth and large chromatic number after discovering that, in graph theory, probabilistic methods are effective in addressing extremal problems (Bollobás 2001; Frieze and Karoński 2016). The theory of random graphs, thus, lies at the crossroads of probability theory and graph theory (Hatano and Mesbahi 2005). By and large, complex networks have distinctive empirical properties and they grow in an unpredictable manner. From social networks to the World Wide Web to biological networks, challenges are faced in understanding their structure and development. The theory of random graphs is widely used to model and analyze most complex networks for studying their behavior and for capturing the uncertainty and the lack of regularity. Over the past two decades, a volume of random graph models have been developed to capture the behavior of small-world and scale-free complex networks.

There are a number of books on the multidisciplinary field of random graphs. Properties of the real-world networks, their models and dynamical processes living on them are partly covered in Janson et al. (2000), Bollobás (2001), Marchette (2004), Durrett (2007), Newman (2010), Frieze and Karoński (2016) and Krivelevich et al. (2016). However, until now, there has been no comprehensive book specifically dealing with random graph models for real-world networks. The book by Remco van der Hofstad fills this gap by studying random graphs as models for complex networks, summarizing the insights 
developed in the quest for the recent advances and placing the developments in a unified framework to facilitate a systematic study.

\section{Book review}

The book is organized into an introductory chapter and three other parts. The first chapter introduces complex networks, their empirical properties and the kinds of random graph models investigated in the book in order to form an inspiration for the subsequent parts. The first part gives the basic probabilistic tools used in the later parts and describes branching processes. The second part covers the classical Erdôs-Rényi random graph and the third part discusses advanced models for complex real-world networks.

\section{Part I}

The second chapter describes basic results in probability theory and discusses convergence of random variables, large deviations, martingales, coupling, extreme value theory and the first and second moment methods. The third chapter describes branching processes with special attention to Poisson offspring distribution and their relationship with binomial offspring distribution.

\section{Part II}

Chapter 4 studies the Erdôs-Rényi random graph. Its phase transition and connected components are described in terms of branching processes. The chapter also proves that the largest connected component exhibits a phase transition. Chapter 5 further investigates various other characteristics of the Erdős-Rényi random graph while focusing on its critical behavior, connectivity threshold, and degree structure.

\section{Part III}

Chapter 6 studies inhomogeneous and generalized random graphs. Chapter 7 investigates graphs with fixed degrees and draws associations between the generalized random graphs described in Chapter 6 and the configuration model. Chapter 8 investigates preferential attachment models that describe networks where the numbers of edges and vertices grow linearly with time. The chapter also shows the behavior of the proportion of vertices with a fixed degree as a function of time.

\section{Price}

The book is available in hardback in an easily affordable price.

\section{Critical comments}

What makes the book particularly interesting is that it provides all important preliminaries for readers not having the basic background knowledge of random graphs. However, the book does not include necessary proofs of the preliminary results and only provides pointers to the literature. Moreover, as the book is intended for a graduate course, it would have been useful for students and instructors to have had the book accompanied with online or on-disk supplementary material, such as lecture slides, sample exams with solutions and learning assessments. 


\section{Conclusion}

The book is well-suited for a graduate course on random graphs, where students may only have minimal background in probability theory, as the book provides plenty of motivation for the topic and covers all important preliminaries. All the chapters are supplemented by extensive exercises to develop better intuition and to progressively master the models covered in the book. In a nutshell, the book is easy to follow and well-organized for developing proficiency in random graph models necessary for state of the art research.

\section{Authors' information}

Ghulam Abbas received his B.S. degree in computer science from the University of Peshawar, Pakistan, in 2003, and his M.S. degree in distributed systems and Ph.D. degree in computer networks from the University of Liverpool, U.K., in 2005 and 2010, respectively. From 2006 to 2010, he was a Research Associate with Liverpool Hope University, U.K., where he was associated with the Intelligent \& Distributed Systems Laboratory. Since 2011, he has been with GIK Institute of Engineering Sciences and Technology, Pakistan. Currently, he is serving as Associate Professor. Dr. Abbas is a Fellow of the Institute of Science \& Technology, U.K., a Fellow of the British Computer Society, and a Senior Member of the IEEE. His research interests include complex networks, wireless communications and distributed systems.

\section{Competing interests}

The author declares no competing interests.

\section{Publisher's Note}

Springer Nature remains neutral with regard to jurisdictional claims in published maps and institutional affiliations.

Received: 6 October 2017 Revised: 16 October 2017 Accepted: 19 October 2017

Published online: 27 October 2017

\section{References}

Bollobás B (2001) Random graphs, 2nd edn. Cambridge Studies in Advanced Mathematics. Cambridge University Press, Cambridge

Durrett R (2007) Random graph dynamics. Cambridge Series in Statistical and Probabilistic Mathematics. Cambridge University Press, Cambridge

Erdős P, Rényi A (1960) On the evolution of random graphs. Publications of the Mathematical Institute of the Hungarian Academy of Sciences 5:17-61

Frieze A, Karoński M (2016) Introduction to random graphs. Cambridge University Press, Cambridge

Hatano Y, Mesbahi M (2005) Agreement over random networks. IEEE Trans Autom Control 50(11):1867-1872

Janson S, LuczakT, Rucinski A (2000) Random graphs. Wiley-interscience series in discrete mathematics and optimization. Wiley, New York

Krivelevich M, Panagiotou K, Penrose M, McDiarmid C (2016) Random graphs, geometry and asymptotic structure. Cambridge University Press, Cambridge

Marchette DJ (2004) Random graphs for statistical pattern recognition. Wiley, New Jersey

Newman MEJ (2010) Networks: an introduction. Oxford University Press Inc., New York

Newman MEJ, Strogatz SH, Watts DJ (2001) Random graphs with arbitrary degree distributions and their applications. Phys Rev E 64(026118):1-17 